\title{
A dichotomy in dust-jet orientation in radio galaxies
}

\author{
Gijs Verdoes Kleijn ${ }^{1}$ and Tim de Zeeuw ${ }^{2}$ \\ ${ }^{1}$ ESO, Karl-Schwarzschild-straße 2, Garching, 85748, Germany email: gverdoes@eso.org \\ ${ }^{2}$ Leiden Observatory, Postbus 9513, NL-2300 RA, Leiden, Netherlands
}

\begin{abstract}
We have analyzed the position angle (PA) differences between radio jets and dust distributions in the centers of Fanaroff \& Riley Type 1 (FRI) radio galaxies. We model the observed PA differences to infer the three-dimensional relative orientation of jet and dust. Our main conclusion is that there is a dichotomy in dust-jet-galaxy orientation both in projection and in three-dimensional space. The orientation dichotomy can explain the contradictory results obtained in previous studies. We briefly mention scenarios that might explain the dichotomy.
\end{abstract}

The relative orientations of the jets, dust and stellar host pose constraints on the physical processes that govern the jet orientation and perhaps the jet formation mechanism in radio galaxies. Many studies have found that jets in radio galaxies are roughly perpendicular to the dust structures (e.g., Kotanyi \& Ekers 1979; Möllenhoff, Hummel \& Bender 1992; van Dokkum \& Franx 1995; Verdoes Kleijn et al. 1999; de Koff et al. 2000; de Ruiter et al. 2002; Capetti \& Celotti 1999; Sparks et al. 2000). In contrast, Schmitt et al. (2002) found for a sample of 20 radio galaxies with regular dust disks that the jets are not at all perpendicular to the disks in three-dimensional space.

We have compiled a sample of $37 \mathrm{FR}$-I dusty radio galaxies at $z<0.15$, with published radio-jet orientations, for which we could find HST archival imaging to determine the intrinsic relative orientation of (ir)regular dust, jet and stellar host.

We classify the dust morphology in three bins of increasing irregularity. There are 17 'ellipses' which have a smooth elliptical circumference with ellipticity $\epsilon, 9$ 'lanes', which are thin filamentary structures with bends and/or mulitple strands. Nine galaxies have a rather regular dust morphology intermediate between ellipse and lane. Lastly, there are 2 'irregular dust' features which are too clumpy and irregular to establish an orientation. We define the dust orientation as the PA of the longest linear axis for lanes, or of the dust major axis for ellipses.

Figure 1a shows a dichotomy in the PA difference between dust and galaxy major axis $\triangle \mathrm{PA}_{\mathrm{DG}}$ as a function of the PA difference between dust and jet axis $\triangle \mathrm{PA}_{\mathrm{DJ}}$. Dust structures which are misaligned with the galaxy $\left(\Delta \mathrm{PA}_{\mathrm{DG}}>20^{\circ}\right)$ are typically 'lanes' and have roughly perpendicular jets $\left(\Delta \mathrm{PA}_{\mathrm{DJ}} \gtrsim 60^{\circ}\right)$. In contrast, the radio jets have no clear preferential direction (i.e., $\Delta \mathrm{PA}_{\mathrm{DJ}} \sim\left[25^{\circ}-90^{\circ}\right]$ ) to dust structures which are aligned with the galaxy $\left(\Delta \mathrm{PA}_{\mathrm{DG}}<20^{\circ}\right)$ which are typically classified as 'ellipses'.

The sky-plane projected properties of the dust-jet system, $\Delta \mathrm{PA}_{\mathrm{DJ}}$ and $\epsilon$, place constraints on the three-dimensional relative orientations. The dust ellipses are consistent with circular thin disks observed at random viewing angles. We therefore model these system as circular disks for which the angle $0^{\circ} \leqslant \theta_{\mathrm{DJ}} \leqslant 90^{\circ}$ between jet-axis and the disk rotation-axis follows a Gaussian distribution. This model gives the simultaneous probability distribution for $\left(\Delta \mathrm{PA}_{\mathrm{DJ}}, \epsilon\right)$ for a given mean $\mu$ and dispersion $\sigma$. Figure $1 \mathrm{~b}$ shows the best-fitting $\mu$ and $\sigma$ for 100 bootstrapping runs using a maximum-likelihood analysis. In contrast to ellipses, we do not know the inclination of dust lanes. The systematic 
(a)

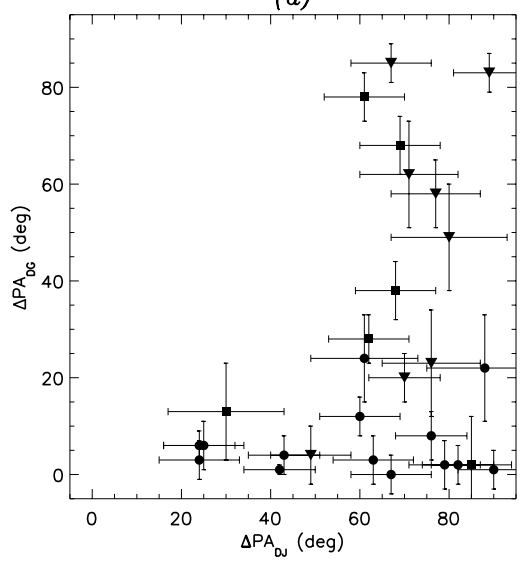

(b)

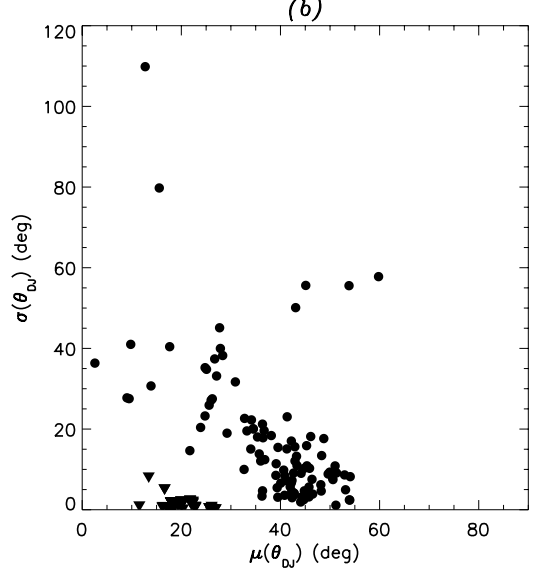

Figure 1. (a):projected PA differences for Dust, Galaxy and Jet. $\triangle \mathrm{PA}_{\mathrm{DG}}$ versus $\Delta \mathrm{PA} \mathrm{DJ}_{\mathrm{J}}$ for dust ellipses (dots), lanes (triangles) and morphologies between ellipse and lane (squares). (b): $3 \mathrm{D}$ misalignment angle $\theta_{\mathrm{DJ}}$ between jet-axis and disk rotation-axis. Best fitting mean $\mu$ versus dispersion $\sigma$ for a truncated Gaussian distribution of $\theta_{\mathrm{DJ}}$ for a 100 bootstrapping runs using a maximum-likelihood analysis for ellipses (dots) and lanes (triangles). Lanes have a narrower $\theta_{\mathrm{DJ}}$ distribution than dust ellipses.

difference in $\triangle \mathrm{PA}_{\mathrm{DG}}$ and morphology argue against the lanes being simply edge-on ellipses. Dust lanes could be perturbed disks, viewed relatively close to edge-on, as has been argued for two dust lanes in the sample, NGC 5128 and M84 (Quillen et al. 1992; Quillen \& Bower 1999). This interpretation requires that face-on lanes are classified differently, i.e., as 'irregular dust' and/or round 'ellipses'. Thus we model lanes as edge-on systems, also because this will yield an upper-limit on the width of their $\theta_{\mathrm{DJ}}$ distribution. Figure $1 \mathrm{~b}$ shows that the dust lanes have systematically smaller $\theta_{\mathrm{DJ}}$ than dust ellipses.

At least two scenarios could qualitatively explain the orientation dichotomy. First, the dust is acquired externally, initially forms a perturbed disk (observed as a dust lane) and finally settles into a flat disk (i.e., a dust ellipse). This scenario would imply that jets form roughly aligned with the initial angular momentum of the $\sim \mathrm{kpc}$-scale dust. Second, it might be that radio-jet induced pressure gradients in the ambient gas force some disks to be roughly perpendicular to the jets (and hence generally misaligned with the galaxy potential), creating disk warps and perturbations in the process (Quillen \& Bower 1999). A full analysis of the radio-jet orientation dichotomy and its cause(s) is in progress.

\section{References}

Capetti A., \& Celotti A. 1999, MNRAS, 304, 434

de Koff S., et al. 2000, ApJS, 129, 33

de Ruiter, H. R., Parma, P., Capetti, A., Fanti, R., \& Morganti, R. 2002, A\&A, 396, 857

Kotanyi C. G., \& Ekers R. D. 1979, A\&A, 73, L1

Möllenhoff C., Hummel E., \& Bender R. 1992, A\&A, 255, 35

Quillen, A. C., de Zeeuw, P. T., Phinney, E. S., \& Phillips, T. G. 1992, ApJ, 391, 121

Quillen, A. C., \& Bower, G. A. 1999, ApJ, 522, 718

Schmitt, H. R., Pringle, J. E., Clarke, C. J., Kinney, \& A. L. 2002, ApJ, 575, 150

Sparks W. B., Baum S. A., Biretta J., Macchetto F. D., \& Martel A. R. 2000, ApJ, 542, 667

van Dokkum, P. G., \& Franx, M. 1995, AJ, 110, 2027

Verdoes Kleijn, G. A., Baum, S. A., de Zeeuw, P. T., \& O’Dea, C. P. 1999, AJ, 118, 2592 